\title{
Criminologie
}

\section{La protection de la jeunesse et le projet de la loi 65}

\section{Michel Lippé}

Volume 8, numéro 1-2, 1975

Délinquance juvénile au Québec

URI : https://id.erudit.org/iderudit/017036ar

DOI : https://doi.org/10.7202/017036ar

Aller au sommaire du numéro

Éditeur(s)

Les Presses de l'Université de Montréal

ISSN

0316-0041 (imprimé)

1492-1367 (numérique)

Découvrir la revue

Citer cet article

Lippé, M. (1975). La protection de la jeunesse et le projet de la loi 65.

Criminologie, 8(1-2), 9-28. https://doi.org/10.7202/017036ar d'utilisation que vous pouvez consulter en ligne.

https://apropos.erudit.org/fr/usagers/politique-dutilisation/ 
LA PROTECTION DE LA JEUNESSE ET LE PROJET DE LOI 65

Michel Lippé 
C'est le 10 novembre 1972 que le ministère de la Justice et le ministère de la Famille et du Bien-Etre social ont présenté à l'Assemblée Nationale le projet de Loi 65 sur la protection de l'enfance. Le texte de la loi était inspiré par le rapport de l'équipe de ces deux ministères et devait tenir compte également des recommandations de la Commission d'enquête sur l'administration de la justice en matière criminelle et pénale, présidée par le juge Y. Prévost.

Il n'en reste pas moins que, face aux oppositions des groupes et organismes divers, la loi a été renvoyée pour étude.

\section{Pourquoi?}

À la veille de la présentation de la nouvelle version, revue et corrigée, il nous semble utile de reprendre ici certaines critiques qui ont été formulées selon le caractère des divers objectifs poursuivis et selon les prises de position de divers groupes, ou organismes.

\section{a) L'enfant, les parents et l'État}

Parmi ces objectifs, un des premiers visait à préciser les relations entre l'enfant, ses parents et l'Etat, en terme de droits et de devoirs. L'Assemblée Nationale n'a pas fait référence à des droits et devoirs particuliers lors de l'adoption, ou des amendements apportés à la Loi de protection de l'enfance et de la jeunesse, ou encore lors de la déposition en première lecture du projet de Loi 65.

Cette situation a cré des litiges, car ce sont les valeurs de ceux qui administrent le système qui servent alors de points de 
repère. Les valeurs ne font pas toujours l'objet d'un consensus et le sort de l'enfant dépend des personnes avec lesquelles il entre en contact, beaucoup plus que du respect de ses droits et devoirs.

En effet, les valeurs auxquelles adhère un individu reflètent souvent la classe sociale à laquelle il appartient. Nous savons que la clientèle des enfants ayant besoin de protection appartient en grande majorité à la classe défavorisée et que, d'autre part, les juges et le personnel de la Cour se recrutent dans une classe sociale plus favorisée. Or, il ne fait pas de doute que ces derniers ont tendance à vouloir imposer leurs vues et leurs standards d'éducation aux enfants et aux parents qui sont dans l'obligation de faire appel à leurs services. Cette situation ne fait que perpétuer l'imposition par les «bien pensants» de valeurs sujettes à caution. La minorité qui impose ses vues à la majorité.

Dix-sept (17) organismes sur vingt-deux (22), qui ont présenté des mémoires, étaient dès lors d'avis qu'une loi de protection de la jeunesse devrait s'appuyer sur une Charte des droits de l'enfant visant à définir ces droits, à préciser les droits et devoirs des parents et les responsabilités de l'État. Dans leur optique, il s'agissait d'une déclaration de certains principes de base devant inspirer ceux qui devront être interprétés et appliqués dans le texte de la loi.

Nous ne pouvons qu'appuyer cette recommandation quasiunanime. Nous parlons tous des droits et des devoirs de l'enfant, des droits et des devoirs des parents à son égard et de l'intervention de l'Etat dans l'exercice de ces droits et devoirs, sans définir de façon claire et précise les droits et devoirs en cause. C'est une situation illogique, puisqu'un système visant à assurer la protection des droits de l'enfant devrait tout d'abord préciser quels sont ces droits et ensuite décider des moyens pour en assurer la protection.

\section{b) Le droit du mineur de faire une demande de protection}

Selon onze (11) organismes, il s'avérait essentiel de prévoir la possibilité pour toute personne raisonnable, même mineure, de recourir au service de protection de la jeunesse. Sans cette possibilité, le mineur, qui devrait pouvoir bénéficier de la protection, serait lui-même incapable de signaler sa situation à la connaissance des autorités. 
Cette recommandation rejoint la première qui vise à reconnaître l'enfant, ou l'adolescent comme un citoyen à part entière. Il doit avoir le pouvoir de se servir d'une loi qui a pour but premier sa protection, d'autant plus qu'il existe de nombreux cas où seul le mineur est au courant des faits et peut réclamer protection pour lui-même.

\section{c) L'ingérence indue de l'État}

Dans le même sens, l'École de Criminologie soulignait que l'enfant et ses parents devraient se voir accorder un recours contre une ingérence indue de l'Etat. Le projet de loi ne prévoyait aucun recours spécial pour l'enfant et ses parents : seuls le S.P.J. et le C.S.S. étant autorisés à requérir l'intervention de la Cour.

Il y a toujours dans ces circonstances la possibilité d'utiliser l'injonction. Cependant, ce moyen est, à notre avis, insuffisant. La complexité de la procédure en Cour supérieure et la nécessité absolue de recourir à un avocat le rendent, à toutes fins utiles, peu accessible. Alors qu'un recours spécial à la Cour de BienEtre Social en raison de la souplesse de la procédure et la nonnécessité de recourir à un avocat, serait plus facilement accessible aux citoyens de toutes les classes sociales.

Cette recommandation va dans le sens de la première et met en lumière l'absence d'une Déclaration des droits de l'enfant. La Déclaration canadienne des droits de l'homme reconnaît à tout citoyen canadien le droit de requérir l'intervention des tribunaux contre toute ingérence, jugée indue, de l'État dans l'exercice de ses droits et devoirs. Pourquoi l'enfant et ses parents ne peuventils disposer du même recours ?

La nécessité d'une déclaration officielle des droits de l'enfant (et son corollaire : droits et devoirs des parents et de l'État) est essentielle et prioritaire, principalement dans le contexte de la "protection assortie de contrainte ». La possibilité que les valeurs personnelles de ceux qui prennent les décisions joue n'est pas le meilleur moyen d'éviter l'arbitraire. Ce prérequis est une condition de base pour la mise en place d'un système cohérent. Le rappel des principes de la Charte des droits de l'enfant n'est pas suffisant. Cette dernière précise les conditions minimales de vie pour chaque enfant. Une société, comme la nôtre, doit viser plus 
haut : de là la nécessité d'une déclaration officielle de l'Assemblée Nationale des droits de l'enfant québécois.

\section{CRITĖRES RÉGISSANT LE CHOIX \\ DES CIRCONSTANCES DANS LESQUELLES \\ L'ÉTAT EST JUSTIFIÉ D'INTERVENIR}

Nous croyons qu'il est important que le législateur délimite clairement les raisons qui justifient l'intervention de l'État dans la vie privée de l'enfant et de ses parents. Ce principe a été réaffirmé par l'Organisation des Nations Unies, ainsi que par l'Union internationale de la protection de l'enfance'.

Dans cette perspective, l'article $4 \mathrm{du}$ projet de loi utilise des concepts qui laissent place à l'arbitraire. Malgré l'effort du législateur pour mieux définir les critères régissant l'intervention de l'État, il n'en demeure pas moins que les concepts utilisés ne font pas, quant à leur définition, l'objet d'un grand consensus de la part de ceux qui s'intéressent à cette question. Le concept de l'enfant caractériel illustre bien la variété de définitions auxquelles les divers spécialistes font appel.

L'énumération, présentée à l'article 4, est, selon huit (8) organismes, trop limitative. D'après plusieurs, il est nécessaire d'avoir une liste complète des motifs souhaitables d'intervention ou encore d'édicter une clause générale telle "pour toute autre cause jugée suffisante 3 .

Nous ne partageons aucun de ces deux points de vue. Nous sommes persuadés qu'il est impossible de cerner toutes les situations où un enfant a besoin de protection. La réalité dans ce domaine comme dans d'autres, est mouvante et difficile à circonscrire. Par ailleurs, laisser à l'arbitraire des individus le pouvoir de décider dans le contexte de "toute autre cause jugée suffisante » est inacceptable. L'article 15 de la Loi de protection de la jeunesse est un article qui laisse au juge toute discrétion pour accorder protection à l'enfant. Nous avons constaté que ces derniers sont avant tout guidés par leurs propres valeurs. Des juges sont plus complaisants lorsqu'il est question de sexe, de religion ou autres facteurs auxquels ils attachent beaucoup d'importance.

1. La protection de l'enfant $*$ Revue internationale de l'enfant, Genève, octobre 1972. 
Nous croyons que, plutôt que de poser le problème en termes de danger moral et / ou physique, il serait nécessaire de faire un recours à une Déclaration québécoise des droits de l'enfant. Sans cela, ce sont les valeurs des individus, détenteurs des pouvoirs, qui sont les points de référence officiels. Nous n'allons pas jusqu'à croire que dans le cadre de notre recommandation il n'y aurait pas place à un certain arbitraire. Le lien entre les droits officiels et une situation concrète laisse place à interprétation. Cependant, notre proposition aurait le mérite de tenir compte de la fragilité des concepts que le projet de loi met de l'avant pour privilégier le principe de l'intervention de l'État dans la vie privée de l'enfant et de ses parents lorsque l'exercice des droits et devoirs, connu de tous, met en danger l'existence de l'enfant.

Ce point de vue donne plus de poids à la recommandation première sur la question d'une Déclaration officielle des droits de l'enfant québécois. Sans cela, nous ne croyons pas que le système proposé par le gouvernement pour assurer, à l'aide de contrainte, la protection de l'enfant, puisse atteindre en toute équité l'objectif fixé. Il s'agit de la limitation ou de la suspension des droits de l'enfant et / ou de ses parents lorsque l'exercice de ces droits va à l'encontre du bien de l'enfant et que la coopération libre des intéressés ne peut être obtenue. Les critères régissant l'intervention de l'État découlent donc d'une reconnaissance par l'Etat de droits et de devoirs. Sans cela, il n'y a pas de point de repère et c'est l'arbitraire qui prime. C'est dire que le respect des droits des intéressés ne peut être assuré, puisque ces droits ne sont pas connus par l'une des parties impliquées, l'autre étant juge et partie.

\section{PROCEDURE}

Afin d'assurer, en toute équité, le respect des droits des personnes en cause, il est essentiel que la procédure à suivre soit suffisamment formelle. Les mémoires n'ont pas discuté de cette question dans tous ses aspects. C'est en quelque sorte sur des points précis de procédure que l'on retrouve un certain consensus. L'École de Criminologie a cependant recommandé que les standards du \& due process of law» soient retenus comme critères dans l'élaboration de la procédure à suivre. Le Barreau, pour sa part, était du même avis, tout en nuançant quelque peu. Il suggérait le texte suivant : "Les règles de procédure contenues au 
Code de procédure civile s'appliquent aux auditions tenues par la Cour en autant qu'elles ne sont pas incompatibles avec les dispositions de la présente loi ${ }^{2}$.,

Ce point de vue nous paraît réaliste. La nature même du débat devant la Cour, la présence et le témoignage de l'enfant et celui des parents, sont souvent complexes et touchent des points où les liens affectifs sont en cause. Les standards du «due process of law » sont sans doute à retenir. Cependant, un article devrait laisser à la Cour une certaine latitude pour ajuster la procédure lorsque le témoignage et / ou les sentiments de l'enfant le nécessitent. Le juge devrait, néanmoins, motiver par écrit sa décision de faire une entorse à la procédure, lorsqu'il juge opportun de le faire. Ainsi, lors d'un appel, cette décision pourrait faire l'objet de considérations appropriées.

Pour mieux saisir le sens de cette opinion, examinons les divers points de procédure en ce qui regarde le besoin de protection et la décision quant à la mesure de protection.

\section{A. QUANT AU BESOIN DE PROTECTION}

\section{LES PERSONNES QUALIFIÉES}

La question de savoir qui devrait pouvoir être informateur est délicate. Laisser à toute personne majeure la responsabilité d'informer les autorités qu'un enfant est exposé à des dangers moraux et / ou physiques équivaut à permettre à tout voisin de pouvoir, à la suite d'une dispute, dénoncer son antagoniste.

Nous sommes d'opinion qu'il y a lieu de distinguer entre le fait d'informer et l'obligation d'informer. De l'avis de neuf (9) organismes, le projet de loi devrait contenir une disposition protégeant l'informateur de bonne foi contre des recours au civil et / ou au criminel. Il faut noter que si le déclarant n'est pas protégé contre des poursuites possibles, ceci peut conduire des personnes à s'abstenir de faire une déclaration et ainsi, le souci d'éviter les poursuites risque de limiter, ou d'empêcher, les interventions nécessaires. De plus, les citoyens et les spécialistes du

2. Barreau du Québec. Mémoire présenté à la Commission parlementaire sur le projet de loi sur la protection de la jeunesse. Janvier 1973, p. 22. Le Barreau a rendu ce document public avant de comparaître devant la Commission parlementaire. 
domaine peuvent avoir d'excellentes raisons de ne pas rapporter aux autorités une situation de danger pour l'enfant.

Nous croyons qu'il y a lieu également de nuancer l'obligation d'informer. Cette obligation n'existerait que dans les cas où une personne «a des raisons graves de croire». Ainsi, le caractère des circonstances deviendrait le critère dans le cas de l'obligation de dénoncer. Il serait difficile de laisser aux personnes le choix dans les situations graves, d'autant plus qu'il y va de la vie d'un enfant et de la responsabilité collective quant à la protection de l'enfance. Le recours au critère de «bonne foi 》 pour assurer l'immunité de l'informateur nous apparaît être, dans les circonstances, le moyen le plus approprié d'éviter les abus. Ainsi, une personne qui fait une déclaration de bonne foi serait protégée de toute poursuite. Le projet de loi ne faisait aucune mention de l'immunité de l'informateur et de l'obligation d'informer, il y a donc lieu d'y inclure les articles qui traiteront de ces deux questions.

\section{LES PERSONNES ET / OU ORGANISMES MANDATÉS}

Le projet de loi mandate le C.S.S. ou le nouveau service de protection de la jeunesse pour entendre les plaintes ou requêtes et pour procéder à une analyse. En fait, le législateur a voulu s'assurer que la « protection non-assortie de contrainte > soit dans tous les cas considérée d'une façon non équivoque, afin de ne recourir à la contrainte que dans les situations où cela s'avère essentiel pour assurer la protection d'un enfant. Le législateur a apporté une réponse claire à la protection de l'enfance en privilégiant la «protection non-assortie de contrainte » comparativement à celle qui fait appel à la contrainte. En effet, la Commission Prévost, à la suite de la Commission Katzenbach, a posé d'une façon, qui nous apparaît bien réaliste, le problème de l'organisme mandaté en affirmant : "Faut-il équiper le tribunal pour enfant d'un plus grand nombre d'options ou faut-il trouver un substitut au tribunal lui-même ${ }^{3}$. ,

En créant le service de protection de la jeunesse, le législateur a donc opté, partiellement, pour le substitut au tribunal.

Cependant, ce service relève du ministère de la Justice. Le Dr Maurice Gauthier a affirmé explicitement, dans une entre-

3. Commission d'enquête sur l'administration de la justice en matière criminelle et pénale au Québec, vol. 4, t. I. La Cour du Bien-Etre Social, p. 94. 
vue, qu'il doit être à la disposition de la Cour. C'est en quelque sorte une demi-mesure. On retire d'une main une bonne partie de ce qu'on avait donné de l'autre.

Nous croyons en la nécessité de trouver un substitut au tribunal. Actuellement, le tribunal est saisi de tout et de rien, puisque l'accessibilité aux services sociaux et de santé n'est pas assurée. En effet, les services de santé et les services sociaux sont, quant à leur accessibilité, la responsabilité exclusive des professionnels. Or, les clients, qui ne collaborent pas comme le voudrait le professionnel, font l'objet d'une certaine discrimination et se voient même refuser les services auxquels ils ont droit. Il est facile de comprendre que cette situation est encore plus flagrante dans le cas d'enfants exposés à des dangers moraux et / ou physiques, alors que la collaboration de l'enfant et / ou des parents est difficile à obtenir. La Cour du Bien-Ettre Social est souvent le seul organisme qui ne peut refuser d'intervenir, même s'il est conscient que la solution au problème présenté n'est en aucune façon la responsabilité du pouvoir judiciaire. Nous ne voyons pas la nécessité de la création d'un service étatique qui, en parallèle et via le C.S.S., verrait à assurer aux citoyens toute l'assistance nécessaire sur une base de coopération. Le parallélisme, la nécessité de recourir aux services du C.S.S. et le caractère étatique, sont des handicaps insurmontables. De plus, le fait de dépendre du ministère de la Justice est fort paradoxal lorsque l'on aftirme que c'est la responsabilité de ce ministère de voir aux questions où l'usage de contrainte est en cause.

Ces critiques nous amènent à constater qu'en ce qui regarde la gestion des organismes mandatés, le projet de loi était déjà en retard par rapport au progrès qu'on avait apporté par la Loi sur les services de santé et les services sociaux ${ }^{4}$.

Le service de protection de la jeunesse n'a pas du tout le caractère d'un comité où les citoyens assument la responsabilité première de la bonne marche de l'organisme. La Loi sur les services de santé et les services sociaux vise à faire participer les citoyens à la gestion des services de santé et des services sociaux. Ces buts sont réalisés, entre autres, par les centres locaux de services communautaires, facilement accessibles aux citoyens ayant besoin de services, et par l'élection du conseil d'administration. Le C.S.S., pour sa part, sera soumis aux mêmes conditions quant

4. 1971, chap. 48. 
aux membres de son conseil d'administration et aura pour mission d'offrir, dans un contexte d'accessibilité, des services sociaux spécialisés.

Nous sommes d'opinion qu'il est nécessaire de confier aux organismes créés par la Loi sur les services de santé et les services sociaux les tâches que le projet de loi 65 voulait confier au service de protection de la jeunesse. Il nous paraît inutile, coûteux et inefficace de créer un service parallèle alors que l'on est en train de mettre sur pied un système de distribution de services de santé et de services sociaux. Car l'existence de deux réseaux parallèles peut même avoir pour conséquence que l'on ne s'occupera pas des cas les plus insolubles, chaque réseau attribuant naturellement la responsabilité à l'autre. Quatorze (14) organismes ont d'ailleurs exprimé leur accord avec cette position et considèrent rétrograde la création d'un service étatique de protection de la jeunesse.

Dans ce contexte, le centre local de services communautaires aura pour rôle de faire le premier tamisage et en quelque sorte, de dépister les premiers signes d'une situation où l'enfant est en difficulté. C'est à cet organisme que l'informateur s'adresse. Le C.S.S., pour sa part, aura pour rôle d'analyser plus en profondeur cette situation qui lui aura été signalée par le centre local de services communautaires et d'offrir des services spécialisés. Ainsi, le souci du législateur de donner une primauté sans équivoque à la «protection non-assortie de contrainte » serait sauvegardé.

Il est essentiel de souligner que toute décision du C.S.S. serait nécessairement prise avec l'assentiment des parents et de l'enfant. Par ailleurs, le C.S.S. devrait être l'organisme mandaté pour requérir l'intervention de la Cour lorsque l'enfant et / ou ses parents refusent l'aide proposée et jugée essentielle.

Le projet de loi prévoyait cette éventualité pour le C.S.S. et le service de protection de la jeunesse. La contre-partie de cette situation consiste à permettre à l'enfant et / ou à ses parents de requérir l'intervention du C.S.S. La Cour aura alors le pouvoir de contrôler les interventions des agences de l'Etat dans des cas spécifiques où l'exercice des droits des individus est en cause. Notre proposition simplifierait grandement la procédure et éviterait aux citoyens les références d'un organisme à l'autre. Le citoyen s'adresse au centre local, organisme accessible et connu 
pour tout service de santé au service social et alors le mécanisme se met en marche.

\section{L'AVIS D'AUDITION}

Selon trois (3) organismes, il apparaît inutile de prévoir l'expédition d'un avis d'audition ou d'une décision de la Cour à un enfant qui n'est pas d'âge raisonnable. Il est certain qu'il faut encore ici nuancer. Ce qui importe c'est la reconnaissance du principe et à ce sujet, le projet de loi est explicite. En effet, l'article 18 stipule qu'un avis d'audition doit être expédié à l'enfant et à ses parents. On ne fait aucune distinction quant à l'âge de l'enfant.

Nous sommes portés à croire que c'est là la meilleure des positions puisqu'il est souvent difficile de statuer sur l'âge d'un enfant sans consulter son extrait de naissance. Dans le cas de très jeunes enfants, il est vrai qu'ils ne peuvent prendre connaissance de l'avis, cependant les personnes qui en assurent la garde seront ainsi informées.

\section{L'EXPOSÉ DE LA PREUVE}

Lors de l'audition d'une affaire, l'article 19 ne prévoit aucune procédure à suivre. Cet état de choses est très malsain. Nous croyons que la proposition du Barreau, citée antérieurement, trouve tout son sens au moment où la preuve se matérialise. L'ensemble de la procédure doit se rapprocher des standards du "due process of law ». L'établissement de la preuve doit donc, en raison de son importance, faire l'objet d'une procédure suffisamment formelle pour garantir les droits en cause.

L'article 20 établit que les audiences doivent avoir lieu à huis clos. Cela nous apparaît être une arme à deux tranchants. Le huis clos permet d'éviter que des problèmes intimes soient connus de personnes qui n'ont aucune raison d'être informées. Cela est particulièrement vrai dans les petites agglomérations. Par contre, il est nécessaire que des personnes de l'extérieur aient accès aux audiences. Rien n'incite plus les cours à maintenir leurs standards de justice. Nous croyons qu'une formule, tentant de concilier ces deux exigences, devrait être conçue. La Commission Prévost a d'ailleurs recommandé la création d'un comité de la cour ayant pour attribution d'informer le public sur son fonctionnement. Les membres élus du conseil d'administration 
du C.S.S. nous apparaissent tout désignés pour jouer ce rôle. Ils sont élus au plan de la région et sont responsables devant leurs électeurs. Ils sont donc mandatés et l'extension de leur mandat consisterait à représenter le public à la Cour afin de surveiller ce qui s'y passe et de l'informer. Le mode électif rend encore plus pertinent l'exercice de ce rôle.

\section{LES NOTES D'AUDIENCE}

Ce point de procédure est relié au droit d'appel. Il s'agit des notes sténographiques, de l'enregistrement sur bandes magnétiques, qui permettent de suivre le déroulement de la procédure et le mot à mot des divers témoignages. Le projet de loi de même que la Loi de protection de la jeunesse n'en font aucune mention. Dans la perspective de la reconnaissance d'un droit d'appel, il s'avère essentiel que la teneur exacte des témoignages soit enregistrée.

\section{LE DROIT À UN AVOCAT}

Le projet de loi n'en fait aucune mention. Nous croyons que l'on devrait créer l'obligation d'informer les parties qu'elles ont droit d'être représentées par un avocat. Cela, parce que l'un des objectifs principaux d'une loi de protection de la jeunesse est la protection de l'enfant et, qu'en conséquence, il a droit à une défense pleine et entière au niveau de ses intérêts et de sa personne. De plus, le juge devrait lors de la première audience, rappeler aux parents et à l'enfant qu'ils peuvent recourir aux services d'un avocat ou s'en faire désigner un d'office.

\section{LE DROIT D'APPEL}

Le projet de loi ne prévoit aucune forme d'appel d'une décision de la Cour. Il est impensable que l'on ne donne pas le droit d'appel aux parties en cause. C'est là, à notre avis, le fruit de préjugés selon lesquels le tribunal des enfants est une «petite cour 》 qui ne s'occupe que de petites causes. Onze (11) organismes affirment la nécessité d'un droit d'appel. Il est grand temps que l'on admette que les droits des enfants sont aussi importants que ceux des adultes et qu'une protection similaire doive en garantir l'exercice.

Les juges ont le pouvoir d'imposer à un enfant un hébergement de longue durée. Des décisions aussi importantes ne doivent pas être prises sans que les droits de l'enfant et de ses parents 
ne soient protégés par des garanties, tel le droit d'appel. Refuser de telles garanties aux justiciables a pour conséquence d'engendrer chez l'enfant et ses parents des sentiments d'injustice bien compréhensibles.

Nous recommandons donc que l'on reconnaisse explicitement dans la Loi le droit des parents et des enfants d'en appeler d'une décision de la Cour.

\section{B. QUANT À LA MESURE DE PROTECTION}

\section{LE RECOURS À L'EXPERTISE}

Les sept (7) organismes qui ont soulevé cette question n'étaient pas tous d'accord sur la nécessité d'une obligation au recours obligatoire à l'expertise dans le choix d'une mesure de protection. Selon certains, sauf en cas d'urgence et de mesures provisoires, jamais le juge ne devrait prendre de décision sans demander une expertise psycho-sociale. D'autres affirment que la Cour peut le demander si elle le juge utile, mais advenant le cas où un tel rapport est produit, il doit être possible de le contredire.

Nous pensons que le recours à l'expertise devrait être obligatoire. Cependant, nous sommes d'avis qu'il y aurait opportunité de le soumettre à une discussion contradictoire. Cette dernière permettra une discussion rigoureuse du cas à l'étude, contribuera à la sauvegarde des droits des parties en présence et au prononcé d'une sentence mieux adaptée.

Cela peut paraître lourd comme procédure, cependant il faut se rappeler que nous sommes alors dans des situations extrêmes où toutes les autres solutions ont été explorées sans que les parties en présence puissent en venir à une entente.

\section{LES MOTIFS DE DÉCISION}

Le projet de loi, à l'article 23, stipule que la Cour doit donner par écrit les motifs de sa décision et cela dans tous les cas et dans toutes les dispositions. Nous louons l'obligation que le législateur a imposée à la Cour, car c'est là un moyen de s'assurer que les décisions ne seront pas prises à la légère. De plus, cette disposition pourrait permettre, dans le contexte d'un droit d'appel à des instances supérieures, d'exercer un contrôle sur la valeur des décisions renđues. 


\section{MESURES DE PROTECTION ET POUVOIRS DE LA COUR}

En ce qui concerne les mesures de protection, trois situations nous apparaissent devoir être discutées. En effet, le pouvoir de la Cour dans le choix de la mesure peut être limité par une énumération de mesures possibles ou encore être laissé à l'entière discrétion du juge. De plus, le choix de la ressource appropriée et la durée du séjour font l'objet de certaines discussions.

Quinze (15) organismes sont d'opinion que le pouvoir de la Cour doit être limité dans le choix de la mesure de protection, alors que les sept (7) autres sont d'avis contraire. Nous croyons qu'en raison du caractère spécial de la mesure de protection et de la procédure de la discussion contradictoire, il n'est pas nécessaire de limiter le pouvoir de la Cour. L'originalité pourrait alors être une caractéristique des mesures de protection et cela pour le plus grand bien de l'enfant. Le fait de limiter le pouvoir de la Cour est beaucoup plus relié au souci d'éviter les décisions farfelues et les exagérations que d'assurer le lien entre les besoins de l'enfant et la décision à prendre. Or les mesures préconisées plus haut sont de nature à réduire les possibilités de décisions plus étranges qu'originales.

Quant au choix de la ressource appropriée dans les cas d'hébergement obligatoire, le projet de loi en confie la responsabilité au C.S.S. Dans le cadre de la discussion contradictoire, nous croyons que le C.S.S. devrait faire un choix qui ferait l'objet d'une discussion entre les parties impliquées. Par la suite, la décision deviendrait exécutoire. Ainsi, on éviterait l'arbitraire du C.S.S. et on permettrait à l'enfant et à ses parents d'exprimer leurs avis quant à la ressource suggérée. La Cour agirait encore ici à titre d'arbitre.

Quant à la durée du séjour en hébergement obligatoire, l'article 28 spécifie qu'il ne peut être d'une durée supérieure à deux années. Nous acceptons le principe de limiter obligatoirement la durée du séjour en raison de la suspension des droits de l'enfant et de ses parents. Cependant, nous aimerions qu'il soit possible de prolonger ce séjour lorsque le bien de l'enfant le nécessite. Il faut se rappeler qu'en vertu du projet de loi, un enfant de huit (8) ans peut être placé en foyer nourricier, alors qu'il est orphelin de père et de mère, et qu'à dix (10) ans il ne soit pas encore en mesure de voler de ses propres ailes. 
Nous croyons que la prolongation du séjour devrait être tout d'abord soumise à l'exigence d'une nouvelle expertise qui conclurait à la nécessité de la poursuite du placement de l'enfant. Dans le cadre d'une discussion contradictoire devant la Cour, l'expertise ferait l'objet d'un échange de vue par les parties impliquées.

Le recours à la discussion contradictoire nous amène donc à recommander que le législateur laisse toute latitude à la Cour, aux experts, à l'enfant et à ses parents pour en arriver à une solution conforme aux besoins de l'enfant dans le plus grand respect des parties en cause.

De plus, nous croyons qu'une telle limite au sujet de la durée et de la prolongation devrait être présente dans le cas des autres décisions. En effet, la liberté surveillée est une sévère limite à la liberté d'un individu et son usage devrait faire l'objet de sérieuses considérations au même titre que l'hébergement obligatoire.

\section{ORGANISMES COMPÉTENTS :}

\section{POUVOIR JUDICIAIRE ET POUVOIR ADMINISTRATIF}

Le partage des responsabilités, en ce qui a trait à la \& protection assortie de contrainte », nous apparaît devoir se faire entre le pouvoir judiciaire et le pouvoir administratif. En effet, la solution du système à contrôle exclusif du pouvoir judiciaire ne peut en aucune façon répondre aux objectifs de la société québécoise, en raison de la mentalité qui a cours à l'intérieur de cette approche, où tout est axé sur des notions strictes et rigoureuses, relatives à l'administration traditionnelle de la justice. La solution du système à contrôle exclusif du pouvoir administratif, quoique fort séduisante, n'offre pas toutes les garanties que les droits des individus en cause soient protégés selon une procédure suffisamment formelle pour éviter les abus.

La société québécoise est de plus en plus confrontée à des mécanismes complexes de relations entre les individus. Le souci de la garantie de l'exercice des droits des individus a été constant chez plusieurs corps intermédiaires, en particulier la ligue des Droits de l'homme. Cette recherche d'assurer le respect des droits de l'homme va aussi loin que de considérer qu'une expertise psycho-sociale puisse être obligatoire dans le cas où une Cour de justice doit décider du sort d'un justiciable qui a été reconnu 
coupable. Il s'agit donc de faire appel au compromis en s'assurant que les droits de l'enfant québécois puissent, d'une part, être sauvegardés par une procédure suffisamment formelle et, d'autre part, faire l'objet de la meilleure considération possible quant à l'adéquation entre les besoins de service de santé et / ou de service social, et les ressources disponibles.

L'exercice de la «protection assortie de contrainte 》, fait appel, selon le projet de loi, à un système à contrôle partagé entre le pouvoir judiciaire et le pouvoir administratif. Dans l'état actuel des connaissances, cette solution se compare avantageusement avec celle des pays les plus progressifs. De plus, la forte prédominance que le législateur a donnée au pouvoir administratif témoigne du souci d'éviter toute confrontation inutile avec l'appareil judiciaire. Le système proposé se rapproche beaucoup du système français comme l'a écrit Tappan :

L'idéal pour traiter les mésadaptations des enfants serait peut-être une combinaison des vertus des deux systèmes ${ }^{5}$.

Tentons de voir dans quelle mesure le système proposé s'oriente vers cet idéal.

\section{QUANT À LA PRISE DE DECISION}

Nous croyons qu'il est opportun de reconnaitre la juridiction exclusive du juge pour décider d'user de contrainte afin d'assurer la protection d'un enfant. Le débat entre les parents et / ou l'enfant et les services visant à assurer la protection de l'enfance doit nécessairement être tranché par un arbitre et cela dans le plus grand respect des droits des parties en cause. Le tribunal et le juge demeurent toujours, malgré toutes les critiques formulées à leur sujet, l'institution la mieux habilitée pour jouer ce rôle.

Nous sommes en désaccord avec le fait que le législateur ait laissé au juge seul toute latitude pour décider des mesures de protection autres que l'hébergement obligatoire. Autant nous sommes prêts à faire confiance au juge quand il s'agit de trancher des questions de droit et de décider si l'intervention de l'État dans la vie d'un enfant est justifiée, autant nous avons des doutes quant à la compétence du juge quand il s'agit de choisir une mesure particulière pour assurer la protection d'un enfant exposé

5. P. Tappan (1958) * The competent authorities ", Ve Congrès international de défense sociale, Stockholm, Suède. 
à des dangers moraux et / ou physiques. En effet, les juges n'ont généralement aucune formation dans les sciences du comportement qui leur permettrait d'évaluer rigoureusement les problèmes et les besoins d'un enfant. II nous apparaît donc aucunement justifié de confier au juge seul la responsabilité de décider du choix d'une mesure.

C'est dans cet esprit que l'article 26 du projet de loi confie au C.S.S. le choix du centre d'accueil, ou de la famille, lorsque l'hébergement obligatoire doit être ordonné. Cependant, plusieurs décisions (liberté surveillée, le fait de confier l'enfant à une personne de son choix, etc.) aussi importantes sont prises par le juge seul. Il est essentiel que ces décisions soient prises par les personnes les plus aptes à les rendre. Diverses formules peuvent être envisagées : formation obligatoire des juges dans les sciences du comportement, décision collégiale et autres. Nous croyons que la procédure de la discussion contradictoire, discutée plus haut dans notre mémoire, obligerait les parties impliquées (parents et / ou l'enfant, les spécialistes du C.S.S. et le juge) à aller au fond des choses. Cette procédure n'exclut en aucune façon la nécessité de recourir aux personnes les mieux qualifiées dans ces circonstances, par le biais de la décision collégiale, ou autres. Elle permettrait cependant d'éviter aux principaux intéressés (parents et / ou l'enfant) le fait de subir une décision, plutôt que de pouvoir contredire les positions avancées par les spécialistes, ou la cour.

En ce qui a trait à la prise de décision, nous sommes donc d'opinion que le juge seul doit avoir juridiction pour décider d'user de contraintes afin d'assurer la protection d'un enfant. Pour ce qui est de la décision quant à la mesure de protection, nous croyons que la procédure de la discussion contradictoire, en plus de requérir systématiquement l'avis des spécialistes dans toutes les décisions, permettra aux parents et / ou à l'enfant, assistés des spécialistes de leur choix, d'exposer leur point de vue. Le juge aura dans ce contexte un rôle d'arbitre et il nous semble qu'il devra obligatoirement avoir recours à l'avis de spécialistes afin de pouvoir conserver sa crédibilité, puisque les intéressés pourront en appeler de ses décisions.

Treize (13) organismes ont soutenu un partage des responsabilités entre le juge et les spécialistes quant à cette question. Tous reconnaissent la juridiction exclusive du juge en ce qui a 
trait à l'usage de la contrainte. Aucun n'a cependant fait appel à la discussion contradictoire à titre de moyen à privilégier pour assurer la plus parfaite adéquation entre les besoins de l'enfant et les ressources disponibles. Cela ne veut pas nécessairement dire que certains d'entre eux n'auraient pas été d'accord. Is n'en ont tout simplement pas parlé.

\section{QUANT À L'ACTUALISATION DE LA DECISION PRISE}

Faisant suite à notre recommandation quant à la prise de décision, nous estimons, en toute logique, que l'actualisation des mesures de protection devrait relever de l'autorité administrative en raison de la nécessité évidente de la présence des spécialistes des sciences du comportement.

Nous ne croyons pas opportun de mandater le juge pour exercer une supervision sur les activités des organismes administratifs. Nous pensons plutôt que le législateur devrait permettre à l'enfant et / ou à ses parents de requérir l'intervention du juge s'ils croient qu'il y a abus de la part de l'autorité administrative. Cette dernière devrait être en mesure de faire la même chose quand elle considère que l'action des intéressés va à l'encontre du bien de l'enfant. Il n'en demeure pas moins que les activités de l'organisme doivent être supervisées par quelqu'un de l'extérieur qui agirait alors comme chien de garde.

Enfin, le rôle très grand, joué par l'autorité administrative, pose le problème du choix à faire quant au ministère qui assumera cette responsabilité. Le projet de loi postulait une responsabilité bicéphale du S.P.J., du ministère de la Justice, du C.S.S., du Centre d'accueil et du ministère des Affaires Sociales.

Quatorze (14) organismes considéraient comme rétrograde l'appartenance du service de protection de la jeunesse au ministère de la Justice et en réclamaient le rapatriement au ministère des Affaires Sociales. Ils alléguaient que toute situation problème, qui relève de l'application d'une loi de protection de la jeunesse, est reliée d'abord à une problématique psycho-sociale, qui prioritairement doit relever de la responsabilité du ministère des Affaires Sociales, en raison de sa mission particulière.

Nous partageons cet avis pour les mêmes raisons, et aussi parce que l'autorité bicéphale est un handicap sérieux à la bonne 
marche du système. L'expérience de la situation présente est révélatrice à ce sujet. En effet, l'autorité administrative et l'autorité judiciaire constituent dans le système actuel une autorité bicéphale. Les conflits sont nombreux et souvent l'enfant en est la victime. Il est vrai que dans la proposition gouvernementale seule l'autorité administrative est en cause par son partage entre deux ministères. Afin de s'assurer de l'unité de l'action, nous croyons préférable d'identifier le ministère des Affaires Sociales comme seul et unique responsable. D'autant plus que le souci de l'unité d'action est une condition essentielle afin d'assurer la bonne marche d'un système à contrôle partagé entre les deux ordres du pouvoir en cause.

\section{LE PARTAGE DES RESPONSABILITÉS ET LES PERSPECTIVES D'AVENIR}

Désormais le projet de Loi 65 est à l'étude depuis plus d'un an et il est permis de présumer qu'un texte révisé sera présenté au cours de 1974-75. Ce texte sera-t-il plus conforme aux recommandations dont nous avons fait un résumé succinct dans les pages qui précèdent? C'est là la première question qui se pose avec d'autant plus d'acuité d'ailleurs, que les problèmes de protection de l'enfance et de la jeunesse demeurent fort complexes et que dans le système actuel, toujours encore en vigueur, les juges, comme les agents de probation, comme les éducateurs, se heurtent constamment à des difficultés quasi insurmontables.

La deuxième question a trait, par contre, à la révision sousjacente des politiques du Québec, compte tenu de la Loi des Jeunes Délinquants qui est du ressort du gouvernement fédéral. Fait significatif, comme le projet de Loi 65 , le Bill C-192 qui devait remplacer l'ancienne Loi, qui date de 1927, mais qui est toujours en vigueur, a été présenté en Chambre en 1971 et renvoyé au Comité d'étude, à la suite d'une opposition très forte qui a empêché son adoption.

Il semble évident, en somme qu'autant à l'échelle fédérale que québécoise, la collectivité remet en question toute la philosophie du traitement de l'enfance et de la jeunesse malheureuse ou délinquante. Il reste à savoir, toutefois, dans quelle mesure les législateurs parviendront enfin à refléter, à travers le texte de la nouvelle loi, les véritables besoins qui se font jour et dont l'importance s'accentue de plus en plus rapidement. 